International Journal of Automotive and Mechanical Engineering ISSN: 2229-8649 (Print); ISSN: 2180-1606 (Online);

Volume 14, Issue 1 pp. 3938-3953 March 2017

CUniversiti Malaysia Pahang Publishing

DOI: https://doi.org/10.15282/ijame.14.1.2017.10.0320

\title{
Computation of turbulent flow around a square block with standard and modified $k-\varepsilon$ turbulence models
}

\author{
A. Rusdin \\ Department of Civil Engineering, Tadulako University, \\ Jalan Soekarno Hatta Km. 09 Palu 94118, Indonesia \\ *Email: andi.rusdin@untad.ac.id \\ Phone: +62451422611; Fax: +62451422355
}

\begin{abstract}
Two-dimensional Reynold Averaged Navier Stokes (RANS) turbulence model simulations for a flow past a square block with $R_{e}=21400$ are presented in this paper. An available modified $k-\varepsilon$ turbulence model, which is an improved version of the standard $k$ $\varepsilon$ turbulence model, is applied to simulate experiment cases of vortex shedding around a square block. The performance evaluation of the modified model is conducted by assessing its results with the results of the standard $k-\varepsilon$ turbulence model based on the similarity with the experiment measurement data. It is found that the modified model improves the drag coefficient by about $16 \%$, the Strouhal number by about $1.5 \%$, and the length recirculation zone by about factor two compared to that of the standard model. The RMSE values indicate a significant improvement of the time averaged velocity along the centre line by about $59 \%$ and the velocity profile above the square block by about $6 \%$ when the modified model is applied. Generally, the modified $k$ - $\varepsilon$ model indicates advantages compared with the standard $k-\varepsilon$ model. However, discrepancy is found between the model result and the experiment observation for the free stream velocity at downstream and it may be necessary to consider the $3 \mathrm{D}$ effect on turbulent fluctuation in further studies.
\end{abstract}

Keywords: Turbulence modelling; vortex shedding; RANS model; standard $k$ - $\varepsilon$ model.

\section{INTRODUCTION}

The periodical vortex shedding that occurs when a flow passes a square block is greatly relevant in the field of engineering hydraulic design. In engineering practice, the economical and safe design of various structures, such as bridge piers, tower, chimneys, and offshore platforms, which are exposed to fluid flows requires a reliable calculation method for predicting the magnitudes, directions, and frequency of forces on the structure as well as for predicting the velocity fluctuation of the flow around the structure [1]. The task to predict a flow past a structure is not easy to be solved because it encompasses complex phenomena, such as separation and reattachment, unsteady vortex shedding and bimodal behaviour, high turbulence, large-scale turbulent structures, as well as curved shear layers [2]. The accuracy and quality of vortex shedding in the computation modelling of flow past a square body is significantly depend on the turbulent model [3]. Different types of turbulence models have been developed and supported by the rapid development of software and hardware technology. Hence the use of the models in computation fluid dynamics has made it possible to solve many fluid flow application 
problems. Turbulent models, such as the Direct Numerical Simulation (DNS) presented by Speziale et al. [4], Saha et al. [5], Saha et al. [6], Trias et al. [7], and Idris et al. [8], the Reynolds Averaged Naiver-Stokes (RANS) by Bosch and Rodi [3], Saha et al. [9], Shih et al. [10], Wright and Easom [11], Li et al. [12], and Elkhoury [13], and the Large Eddy Simulation (LES), such as works by Murakami and Mochida [14], Rodi [2], Bouris and Bergeles [15], Yu and Kareem [16], Yong et al. [17], Yagmur et al. [18], and Bai et al. [19] are commonly used in engineering applications. The DNS method, which computes all size of eddy motion and does not need a turbulent model to solve the Reynolds stress, is valid only for flow with a low Reynolds number, $R_{e}$, less than $10^{4}$ and requires an enormous number of grid points to capture all scales resolution about $R_{e}{ }^{3}$ [2]; hence, the computation of DNS is uneconomical. For high Reynolds number cases, the LES can be applied to calculate a large scale of vortex shedding, however it cannot compute the smallscale eddies, and as a result, energy is dissipated [20]. The problem can be solved in two ways, firstly by implementing the subgird scale model to define turbulent stresses and secondly by introducing a certain amount of numerical dissipation of energy withdrawal. The treatment of LES can accurately simulate the turbulent flow but is computationally expensive.

The RANS equations are solved to determine the phase-averaged quantity of the flow motion and the superimposed random turbulent fluctuation is simulated with a stochastic turbulent model. Various turbulent models have been developed including the $k$ - $\omega$ turbulence model [21-23], Reynolds stress equation (RSE) model [20], and standard $k-\varepsilon$ turbulence model introduced by Launder and Spalding [24]. The RANS model with the standard $k-\varepsilon$ turbulence model has been widely used for engineering practice because of its computational efficiency and simple discretization [25]. Various engineering applications of computational fluid dynamic have been successfully implemented by using the standard $k-\varepsilon$ turbulence model [26-32]. The model has a fairly good performance for computing boundary layer flows [10, 33-35]. However, Franke and Rodi [36] found that the standard $k-\varepsilon$ turbulence model depicted an unrealistic prediction of the shedding motion due to the overestimate of turbulent kinetic energy in the impinging region. Rodi [2] and Murakami and Mochida [14] have reported that the standard $k$ - $\varepsilon$ turbulence model overestimates the length of the reverse flow zone or the separation zone considerably. Kato and Launder [37] proposed a modification of the $k-\varepsilon$ model by rearranging the turbulent kinetic energy production term. It was reported that the model effectively improved the prediction of kinetic turbulent energy in the stagnation region and the length of the recirculation zone $[2,9,14]$.

The aim of the present study is to assess the performance of the modified $k-\varepsilon$ turbulence model and compare its simulation results with those of the standard $k-\varepsilon$ model. The LDV experiment measurement of turbulent flow around a square block by Lyn et al. [38] with $R e=21,400$, which is a relatively high Reynolds number case, is used as the simulation case. Both the standard and the modified $k-\varepsilon$ turbulence models use an identical numerical domain and a similar set up of boundary conditions. The instantaneous turbulent kinetic energy and vorticity contour plots of both turbulence models are compared to evaluate the vortex shedding generation. The drag coefficient, the shedding frequency, the Strouhal number, and the streamwise and the transverse timeaveraged velocity, as well as the length of the recirculation zone are considered as the basis parameters of the model performance and will be compared with the experiment. 


\section{METHODS AND MATERIALS}

\section{Mathematical Model}

At high Reynolds number flows, the stochastic turbulent fluctuations $\phi^{\prime}$ of the threedimensional flows are superimposed on the periodic unsteady motion $\langle\phi(t)\rangle$ of the vortex shedding. In the wake behind a block square where the vortex shedding occurs, the instantaneous flow component $\phi(t)$ will be detected with a periodic component of the vortex shedding period $T_{p}$ and the turbulent fluctuation component $\phi^{\prime}$ will be characterised with a turbulent fluctuation time scale $T_{t}$. The direct numerical simulation cannot calculate the superimposed stochastic turbulent fluctuation. Large eddy simulations can only directly compute the low frequency part of the stochastic motion and the unresolved high frequency part can be simulated with an additional subgrid scale model. The simulation of LES with an additional model will be very expensive [3]. Figure 1 describes that the instantaneous flow component $\phi(t)$ is the combination of the time mean component $\bar{\phi}$, the periodic component $\tilde{\phi}(t)$, and the turbulent fluctuating component $\phi^{\prime}$. The time mean and the periodic component assemble the averaged component or the phase-averaged $\langle\phi\rangle(t)$.

$$
\phi(t)=\bar{\phi}+\tilde{\phi}(t)+\phi^{\prime}=\langle\phi\rangle(t)+\phi^{\prime}
$$

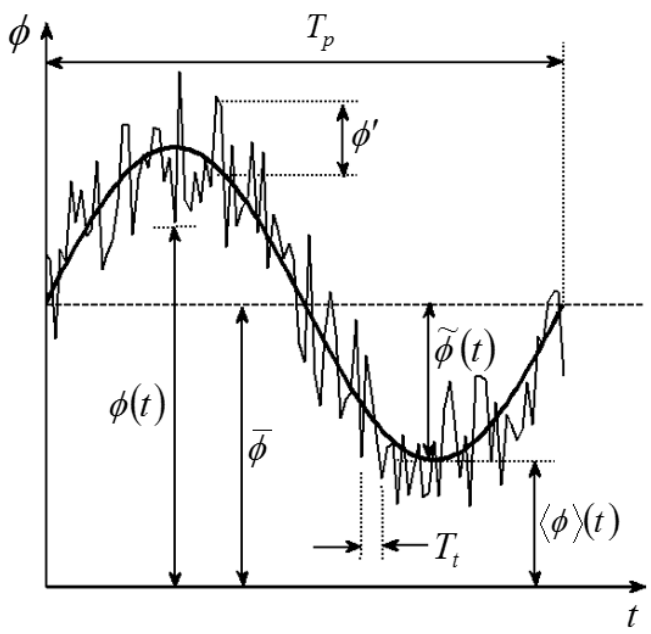

Figure 1. Decomposition of the phase averaged and stochastic turbulent fluctuation components of the vortex shedding flow.

The Reynolds averaged Navier Stokes (RANS) equations resolve the phase averaged velocity and pressure components of the flow past the bluff body and the superimposed stochastic turbulent fluctuating component is computed with a turbulence model. The arrangement of phase-averaged continuity and momentum equations described by Bosch and Rodi [39] can be written as follows:

$$
\frac{\partial\left\langle u_{i}\right\rangle}{\partial x_{i}}=0
$$




$$
\begin{gathered}
\frac{\partial\left\langle u_{i}\right\rangle}{\partial t}+\frac{\partial\left[\left\langle u_{j}\right\rangle\left\langle u_{i}\right\rangle\right]}{\partial x_{j}}=-\frac{1}{\rho} \frac{\partial\langle p\rangle}{\partial x_{i}}+\frac{\partial}{\partial x_{j}}\left[v_{e f f} \frac{\partial\left\langle u_{i}\right\rangle}{\partial x_{j}}-\left\langle u_{i}^{\prime} u_{j}^{\prime}\right\rangle\right] \\
v_{e f f}=v+v_{t}
\end{gathered}
$$

where $t$ is time, $x_{i}$ is the coordinate direction; $\left\langle u_{i}\right\rangle$ is the averaged velocity component, $u_{i}^{\prime}$ is the turbulent velocity, $\langle p\rangle$ is pressure, $\rho$ is the density of the fluid, $v$ is the kinematic viscosity, $v_{t}$ is the turbulent eddy viscosity, and $v_{\text {eff }}$ is the effective viscosity.

The Reynolds stress or the phase averaged products of turbulent velocity fluctuations $\left\langle u_{i}^{\prime} u_{j}^{\prime}\right\rangle$ [37] shown in the momentum equation are calculated with the Boussinesq eddy viscosity approximation.

$$
\left\langle u_{i}^{\prime} u_{j}^{\prime}\right\rangle=v_{t}\left(\frac{\partial\left\langle u_{i}\right\rangle}{\partial x_{j}}+\frac{\partial\left\langle u_{j}\right\rangle}{\partial x_{i}}\right)=\frac{2}{3}\langle k\rangle \delta_{i j}
$$

The kinematic eddy viscosity $v_{t}$ in the standard $k-\varepsilon$ turbulence model is calculated from the following equation.

$$
v_{t}=C_{\mu} \frac{\langle k\rangle^{2}}{\langle\varepsilon\rangle}
$$

Equation (5) shows that the Reynolds stress is related to the kinematic eddy viscosity $v_{t}$ which depends on the turbulent kinetic energy $k$ and the turbulent energy dissipation rate $\varepsilon$. The spatial and the temporal distribution of $\langle k\rangle$ and $\langle\varepsilon\rangle$ values are determined with semi empirical transport equations referred to as the standard $k-\varepsilon$ model introduced by Launder and Spalding [24].

$$
\begin{gathered}
\frac{\partial\langle k\rangle}{\partial t}+\frac{\partial\left[\left\langle u_{i}\right\rangle\langle k\rangle\right]}{\partial x_{i}}=\frac{\partial}{\partial x_{i}}\left[v+\frac{\left\langle v_{t}\right\rangle}{\sigma_{k}} \frac{\partial\langle k\rangle}{\partial x_{i}}\right]+P_{k}-\langle\varepsilon\rangle \\
\frac{\partial\langle\varepsilon\rangle}{\partial t}+\frac{\partial\left[\left\langle u_{i}\right\rangle\langle k\rangle\right]}{\partial x_{i}}=\frac{\partial}{\partial x_{i}}\left[v+\frac{\left\langle v_{t}\right\rangle}{\sigma_{\varepsilon}} \frac{\partial\langle\varepsilon\rangle}{\partial x_{i}}\right]+C_{\varepsilon 1} P_{k} \frac{\langle\varepsilon\rangle}{\langle k\rangle}-C_{\varepsilon 2} \frac{\langle\varepsilon\rangle^{2}}{\langle k\rangle}
\end{gathered}
$$

where the values of the model coefficients are $C_{\mu}=0.09, C_{\varepsilon 1}=1.44, C_{\varepsilon 2}=1.92, \sigma_{k}=1.0$, $\sigma_{\varepsilon}=1.3$. The standard $k-\varepsilon$ turbulence model computes the production term $P_{k}$ in the Eq. (8) as follows:

$$
\begin{gathered}
P_{k}=C_{\mu}\langle\varepsilon\rangle S^{2} \\
S=\frac{\langle k\rangle}{\langle\varepsilon\rangle} \sqrt{\frac{1}{2}\left[\frac{\partial\left\langle u_{i}\right\rangle}{\partial x_{j}}+\frac{\partial\left\langle u_{j}\right\rangle}{\partial x_{i}}\right]^{2}}
\end{gathered}
$$

Franke and Rodi [36] indicate that the standard $k-\varepsilon$ turbulent model over estimate of the turbulent kinetic energy production in the stagnant region is caused by the incorrect 
calculation of the normal stresses in the isotropic eddy viscosity model. Kato and Launder [37] introduced a modification of the production term of $P_{k}$ by replacing one strain stress term $S$ with a vorticity term $\Omega$.

$$
\begin{gathered}
P_{k}=C_{\mu}\langle\varepsilon\rangle S \Omega \\
\Omega=\frac{\langle k\rangle}{\langle\varepsilon\rangle} \sqrt{\frac{1}{2}\left[\frac{\partial\left\langle u_{i}\right\rangle}{\partial x_{j}}-\frac{\partial\left\langle u_{j}\right\rangle}{\partial x_{i}}\right]^{2}}
\end{gathered}
$$

Craft et al. [40] suggested a further modification whereby $C \mu$ depends on the strain rate parameter $S$. The non-constant expression of $C \mu$ indicates that it decreases as the strain rate $S$ increases; however $S$ is restricted to have a maximum value that is equal to 20 .

$$
\begin{gathered}
C_{\mu}=\min \left[0.09, \frac{0.3}{\left(1+0.35 S^{\prime 1.5}\right)}\right] \\
S^{\prime}=\min [20, S]
\end{gathered}
$$

The RANS equations including the turbulence model are discretized with the finite difference written in the CADMAS-SURF code [41]. The method applies the Cartesian staggered grid according to a code developed by Isobe et al. [42]. The NavierStokes Equation is solved with the Simplified Marker and Cell Method (SMAC) by Amsden and Harlow [43] to calculate velocities and the pressure component. The advection and diffusion fluxes are respectively determined with the donor scheme and second order central difference scheme, while the pressure term is evaluated with the forward scheme. The Euler method was applied to solve the time integration of the model.

\section{Computational Setup}

Lyn et al. [38] used a closed water channel and put a square obstacle with diameter $(R)$ that was equal to $4 \mathrm{~cm}$ inside the channel. The LDV was used to record the instantaneous velocity. The constant velocity input, $U_{o}$, was $0.535 \mathrm{~m} / \mathrm{s}$ which gave a Reynolds number $\left(R_{e}=U_{o} R / v\right)$ equal to 21400 .

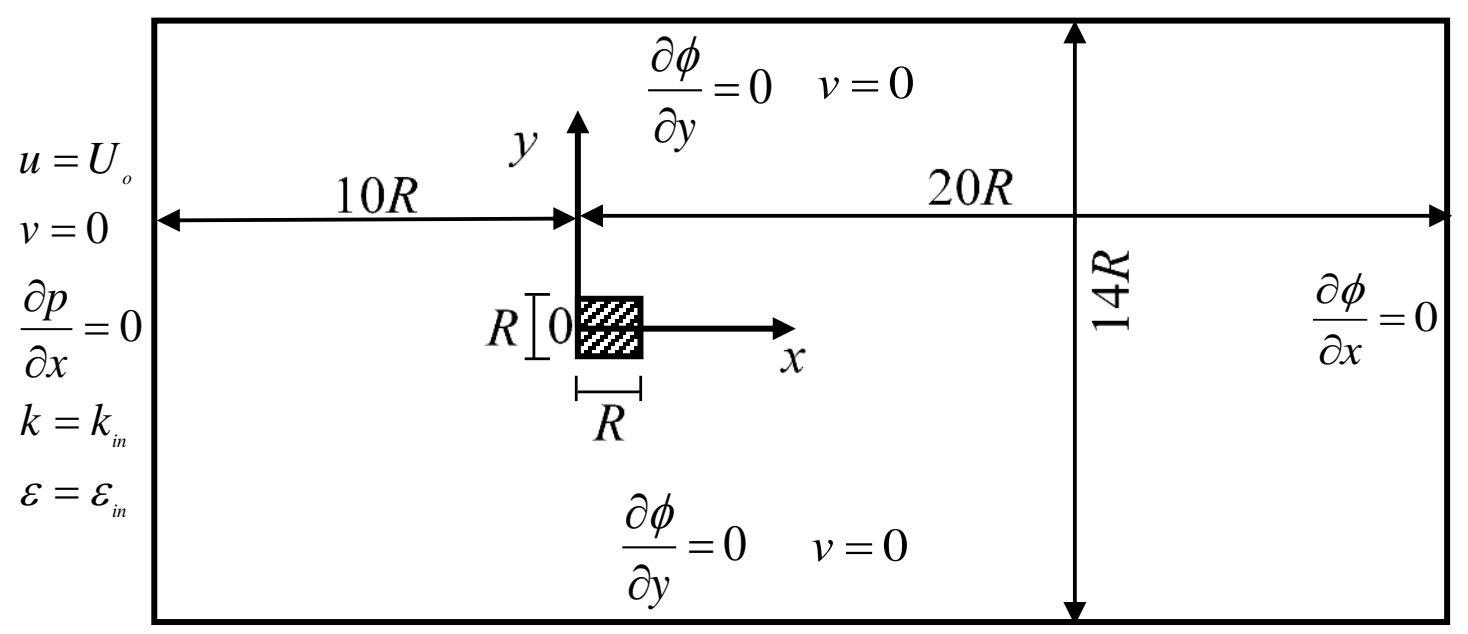

Figure 2. Computational domain. 
The two-dimensional simulation of the flow past a square bluff body is conducted in a domain which has a dimension of $30 R$ in the $x$ direction and $14 R$ in the $y$ direction, see Figure 2. The flow past the square block is a complex flow due to the large-scale periodicity of the separation. The model is expected to give a high accuracy prediction of the complex flow around the obstacle. In this study, the computational domain, which consists of $116 \times 95$ cells, is used to obtain enough accuracy of the complex turbulent flow. The size of the first grid near the wall boundaries was $R / 21$ (about $1.9 \mathrm{~mm}$ ). The expansion criterion of the grid is $0.9 \leq \Delta x_{m} / \Delta x_{m-1} \leq 1.1$, which is suggested by Kimura and Hosoda [25], where $m$ is the grid number. The schematic of the domain can be found in Figure 3.

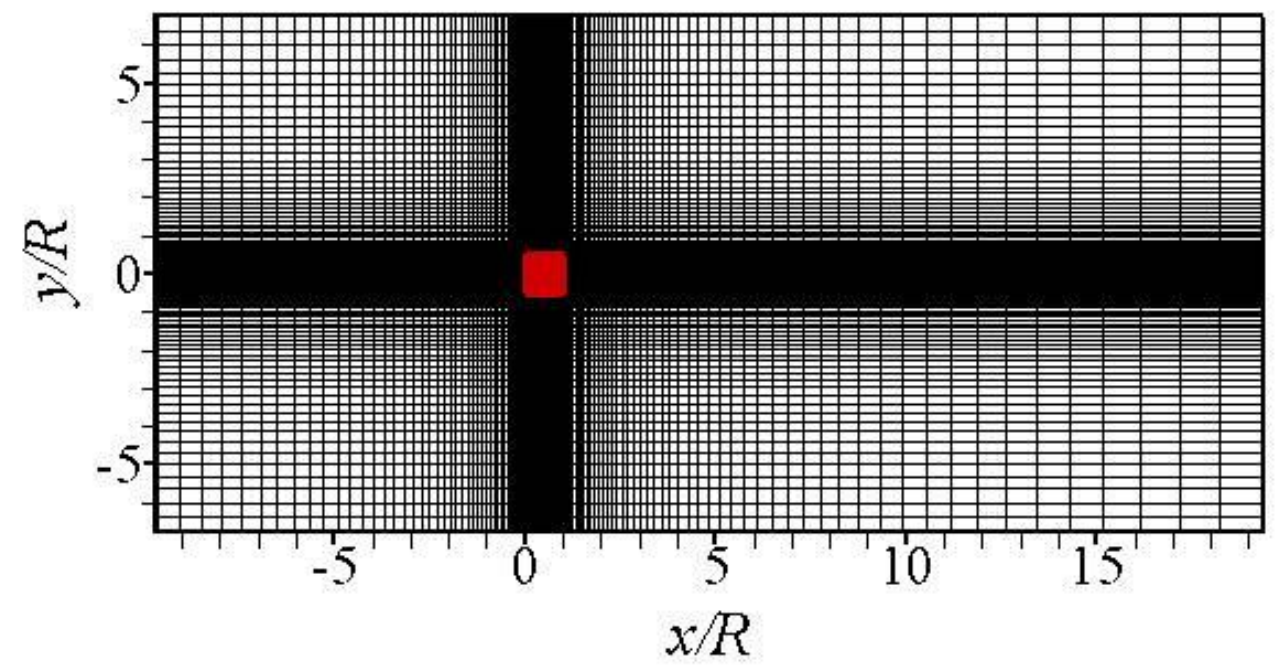

Figure 3. Numerical grid for the flow past a square block.

At the top and bottom of the simulation domain, the slip condition is used for the velocities and the normal gradient that equals to zero is used for $p, k$, and $\varepsilon$. The zero normal gradients are used for the boundary conditions for velocities, pressure, $k$, and $\varepsilon$ at the right boundary. At the surface of the square body, the standard logarithmic law is applied for velocities, the zero-normal gradient is applied for determining the pressure, and the values of $k$ and $\varepsilon$ are determined by the wall function method. The uniform profiles of the streamwise velocity component with $u=U_{o}$ and the transverse velocity component with $v=0$ are applied at the inflow. The level of turbulent intensity, $T_{u}$, at the left domain is $2 \%$ similar with the value used by Lyn [44]. The value of $k$ at the left boundary is determined based on the level of turbulent intensity by Younis and Przulj [1] and calculated by the following equation:

$$
k=\frac{3}{2}\left(u^{\prime}\right)^{2}
$$

where

$$
T_{u}=\frac{u^{\prime}}{U_{o}}
$$

$u^{\prime}$ is the fluctuation velocity component. The inflow magnitude of the dissipation rate, $\varepsilon$, is calculated by using Eq. (6). The ratio of $v_{t} / v=10$ similar with a value applied by Bosch and Rodi [39] is adopted to determine the value of $v_{t}$ at the boundary. 


\section{RESULTS AND DISCUSSION}

Figure 4 depicts the instantaneous turbulence kinetic energy $\langle k\rangle$ around the square block in the case of two different types of turbulence models. The contour plots of $\langle k\rangle$ are not captured in the same angle phase, but they were taken after 60.74 seconds of the simulation time. It can be seen from the figure that the models can give a good representation of the vortex shedding mechanism. The kinetic energy production is vigorous near the wake of the square block where the high gradients of velocity are taking place. It proves that the magnitude of the turbulence kinetic energy depends to the generation of the shear around the square block [9].

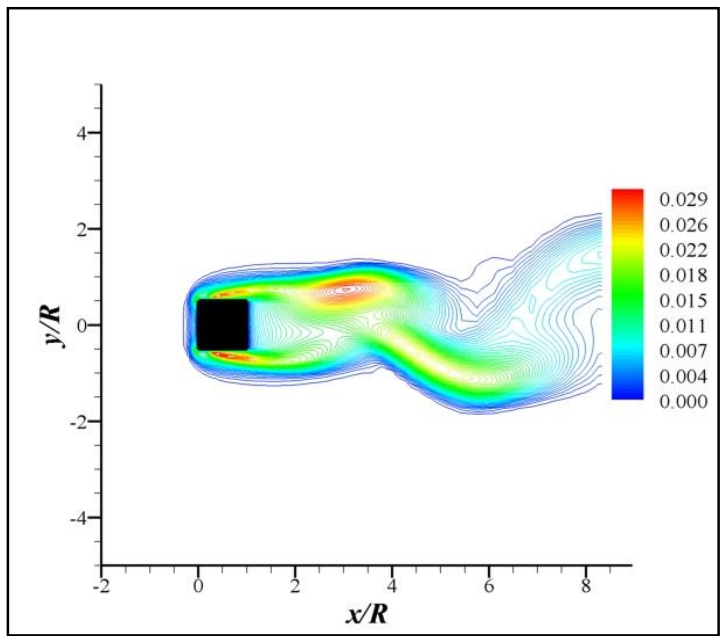

(a)

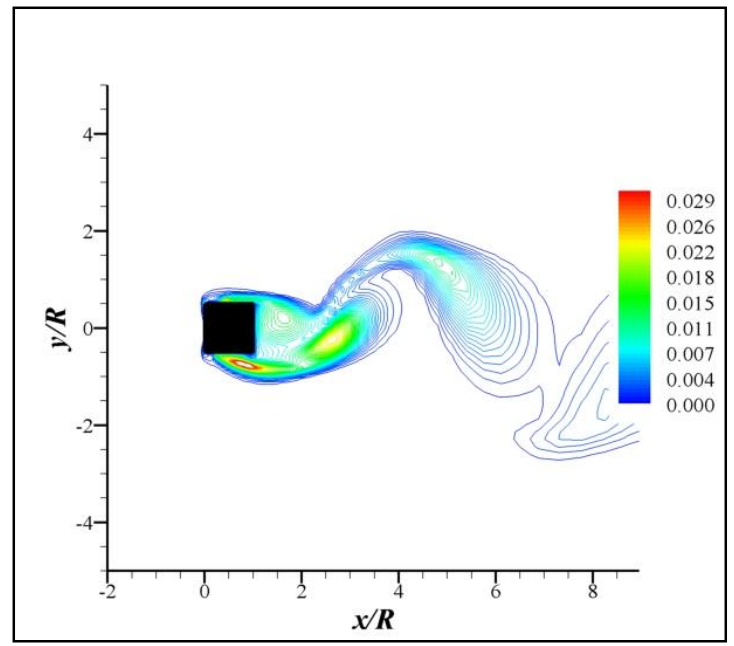

(b)

Figure 4. Instantaneous turbulent kinetic energy, $\langle k\rangle$, (a) Standard $k$ - $\varepsilon$ turbulence model, (b) Modified $k-\varepsilon$ turbulence model

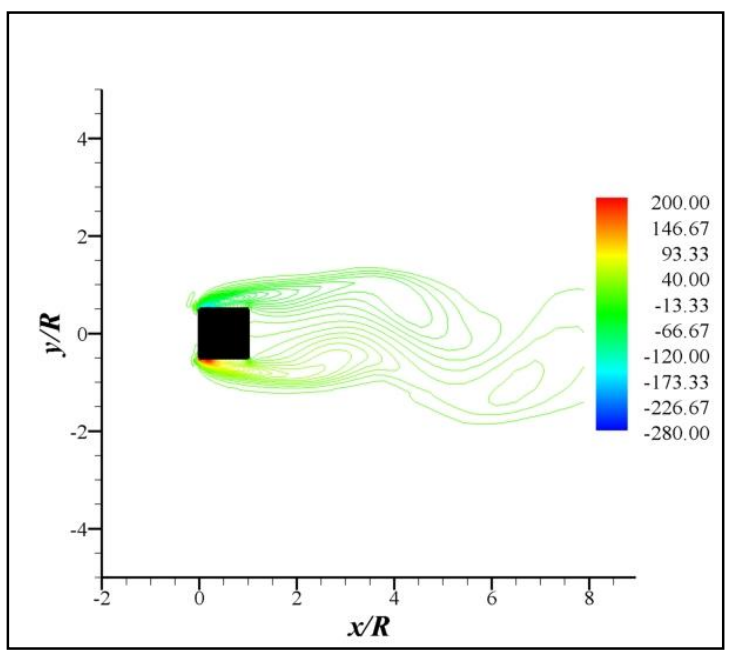

(a)

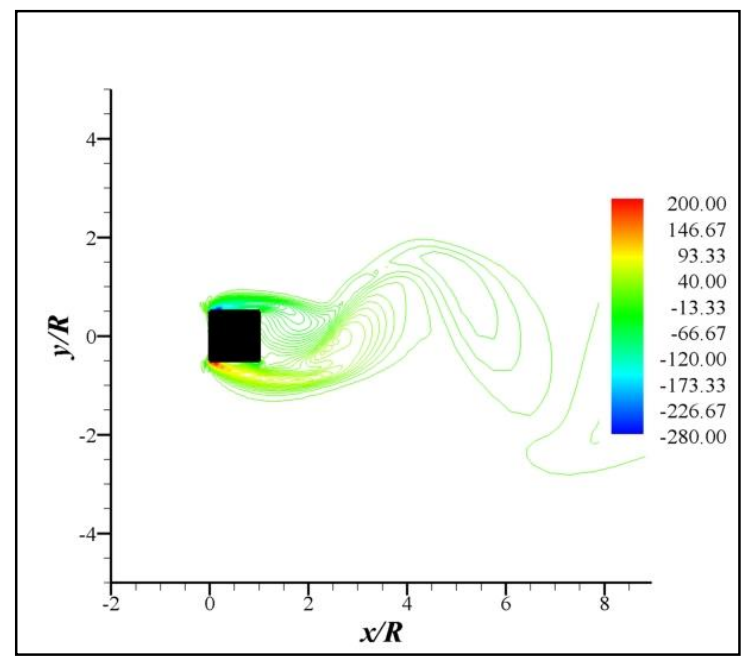

(b)

Figure 5. Instantaneous vorticity contour, $\langle\omega\rangle$. (a) Standard $k-\varepsilon$ turbulence model, (b) Modified $k-\varepsilon$ turbulence model. 
The contour lines indicate that the standard $k-\varepsilon$ model produces excessive turbulence kinetic energy in the vicinity of the stagnant region in front of the square block and the $\langle k\rangle$ pattern in front of the block is corrected when the production term was adjusted in the modified $k-\varepsilon$ model of Kato and Launder [37]. The snapshots of the instantaneous vorticity distributions $(\omega=\partial u / \partial y-\partial v / \partial x)$ near to the square block are presented in Figure 5. Both turbulence models show a correlation of the vorticity fields with the kinetic energy patterns. It seems that the modified $k$ - $\varepsilon$ model generates stronger vortex shedding indicated by an increased number of curvature vorticity contours while the standard model shows slightly long stretched vortices. Bosch and Rodi [3] have reported similar results indicating that the peak of vorticity increases when one strain stress term $S$ is replaced with a vorticity term $\Omega$ for calculating the production term of $P_{k}$.
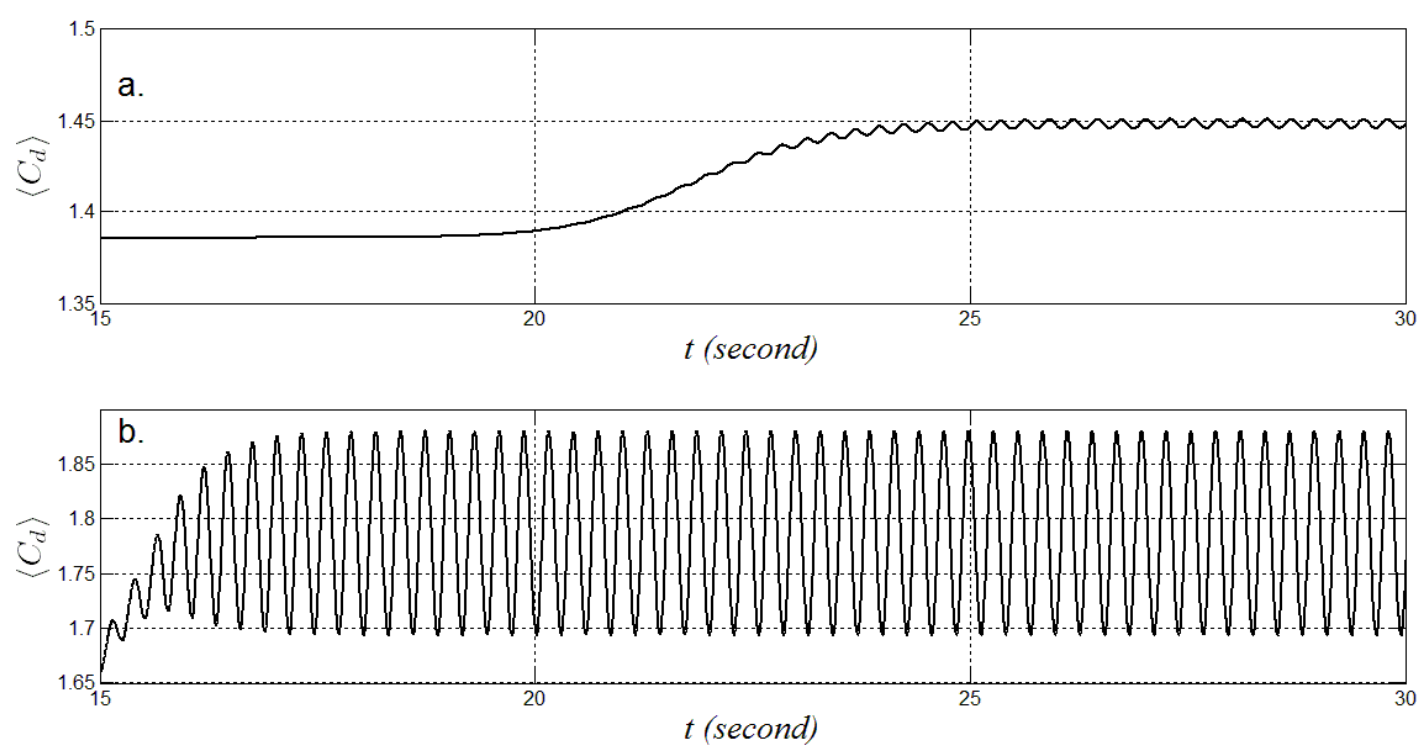

Figure 6. Calculated drag coeficient, $\left\langle C_{d}\right\rangle$, (a) Standard $k-\varepsilon$ turbulence model, (b) Modified $k-\varepsilon$ turbulence model.

The time evolutions of the drag coefficient $\left\langle C_{d}\right\rangle$ computed by the standard $k-\varepsilon$ model and the modified $k-\varepsilon$ model are shown in Figure 6. It seems that the simulations need a spin up time to produce fully developed vortex shedding. The modified $k-\varepsilon$ model evokes the fully developed vortex shedding faster than the standard model. The standard $k-\varepsilon$ model indicates that the vortex shedding is completely formed after about 27.0 seconds from the starting time of the simulation and the modified $k-\varepsilon$ model result shows that the fully developed vortex shedding is formed about 17.0 seconds after the beginning of the simulation. A short spin up time to reach the equilibrium state of turbulent flow has an advantage because it will reduce the simulation time and the computation work becomes inexpensive. Figure 6 pointed out that the fluctuation of the drag coefficient of the modified $k$ - $\varepsilon$ model is more vigorous, where its average value of $\overline{C_{d}}$ is 1.787 and the maximum and minimum values of $\left\langle C_{d}\right\rangle$ are 1.880 and 1.694 , respectively. $\left\langle C_{d}\right\rangle$ of the standard $k-\varepsilon$ model has a small variation where the maximum value of $\left\langle C_{d}\right\rangle$ is equal to 1.451 and the smallest magnitude of $\left\langle C_{d}\right\rangle$ is 1.446 . The mean value of the drag coefficient $\overline{C_{d}}$ of the standard $k-\varepsilon$ turbulent model is 1.448 . Table 1 presents the comparison of the 
vortex shedding parameters of the experiment data and four computation results, i.e. the standard $k-\varepsilon$ turbulence model, modified $k-\varepsilon$ turbulence model, large eddy simulation (LES) model [15], and Reynolds stress equation (RSE) model [20]. The magnitude of the drag coefficient indicates an improvement of about $16 \%$ by the modified $k-\varepsilon$ model compared to that of the standard $k-\varepsilon$ model. Surprisingly, the $\overline{C_{d}}$ of LES and RSE models seem $19 \%$ and $17 \%$ better than the $\overline{C_{d}}$ of the modified $k$ - $\varepsilon$ model and their values are almost similar to the $\overline{C_{d}}$ of the experiment by Lyn [44], see Table 1.

Table 1. Vortex shedding parameters.

\begin{tabular}{lclc}
\hline \multicolumn{1}{c}{ Methods } & $\overline{C_{d}}$ & $f\left(H_{z}\right)$ & $S_{t}$ \\
\hline Experiment [44] & 2.100 & 1.77 & 0.132 \\
Standard $k-\varepsilon$ & 1.448 & 1.80 & 0.134 \\
Modified $k-\varepsilon$ & 1.787 & 1.76 & 0.132 \\
LES [15] & 2.180 & 1.79 & 0.134 \\
RSE [20] & 2.150 & 1.82 & 0.136 \\
\hline
\end{tabular}
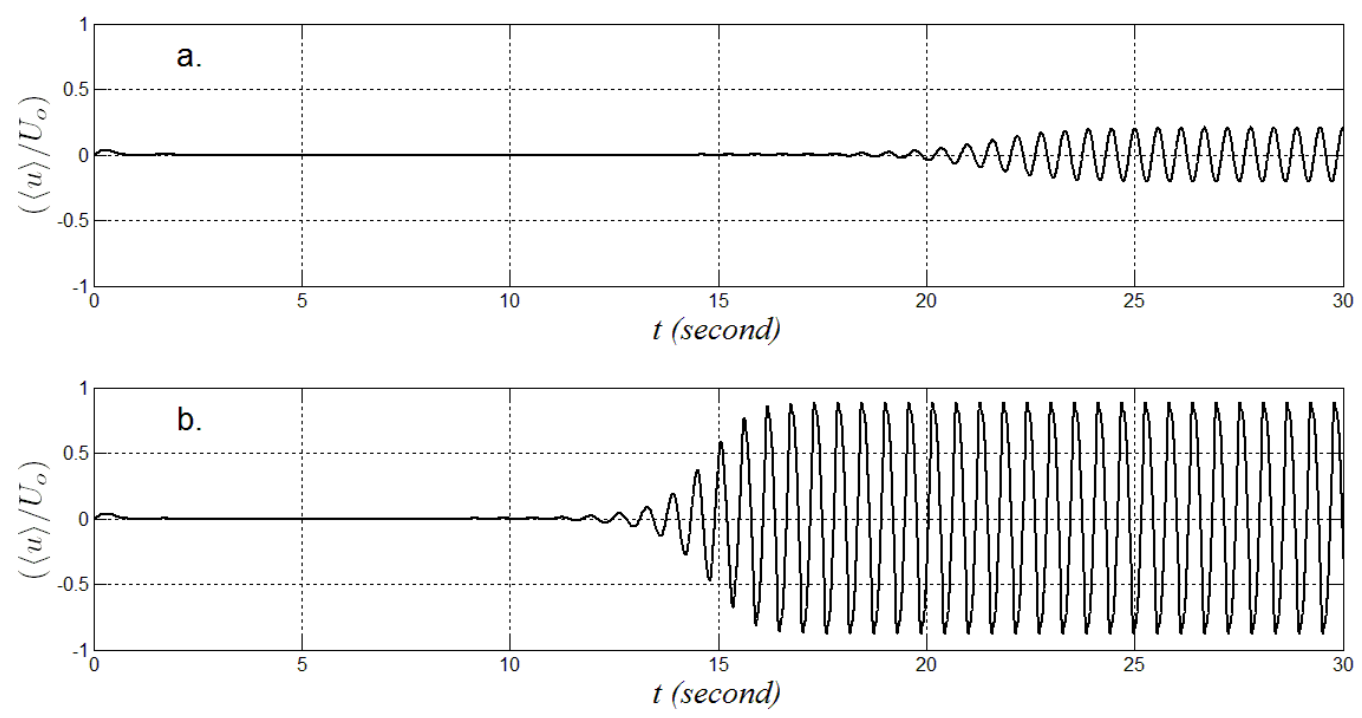

Figure 7. Evolution of the nondimensional transverse component velocity, $\langle v\rangle / U_{o}$, (a) Standard $k-\varepsilon$ turbulence model, (b) Modified $k-\varepsilon$ turbulence model.

The frequency of vortex shedding is calculated by using the $y$ direction velocity $v$ which is recorded at $x / R=2.26$ and $y / R=0.0$. This point is appointed because it is located at the middle of the $y$ direction. Hence, it makes it possible to get a symmetrical time series record of $v$. Figure 7 shows the time series records of $\langle v\rangle / U_{o}$ for both the standard $k-\varepsilon$ turbulence model and the modified turbulence $k-\varepsilon$ model and they indicate symmetrical trends of the time series of the transverse velocities. Figure 8 illustrates that the amplitude of $\langle v\rangle / U_{o}$ obtained from the standard $k-\varepsilon$ model is 0.20 and that of the modified $k-\varepsilon$ model is 0.69 . The standard $k-\varepsilon$ model seems to produce a weak oscillation of $v$. It seems that the incorrect calculation of the turbulent fluctuation by the standard $k$ $\varepsilon$ model propagates low momentum exchanges to the transverse direction [20] and induces the magnitude of the transverse velocity component $v$. In contrast, the modified 
$k-\varepsilon$ turbulence model with the appropriate definition of the production term in the turbulence kinetic energy equation produces a vigorous oscillation of the transverse velocity $v$.
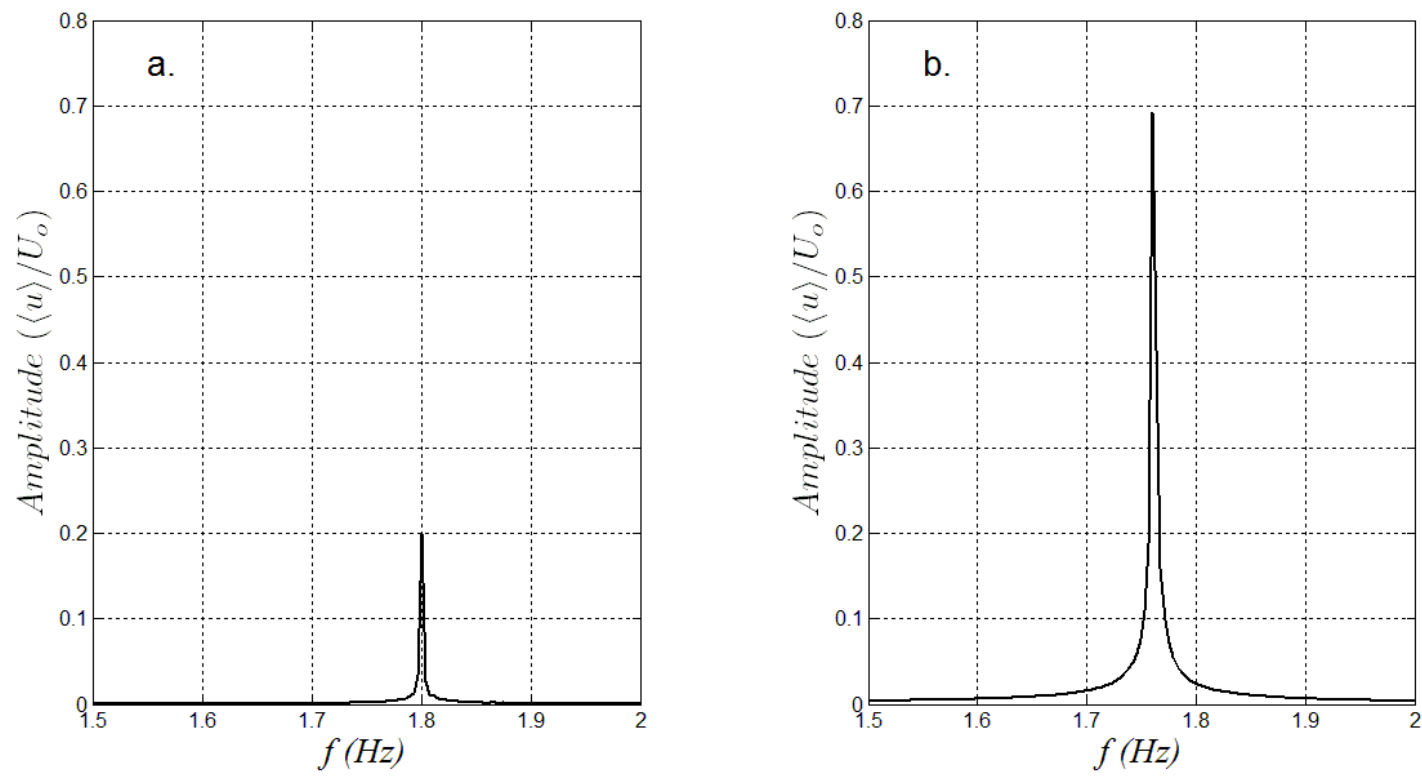

Figure 8. Frequency of vortex shedding calculated from $\langle v\rangle / U_{o}$, (a) Standard $k-\varepsilon$ turbulence model, (b) Modified $k$ - $\varepsilon$ turbulence model.

Figure 8 presents the spectrum of the vortex shedding frequency which can be identified at the maximum value of the amplitude $\langle v\rangle / U_{o}$. The shedding frequency $f$ of the standard $k-\varepsilon$ model is $1.80 \mathrm{H}_{z}$ and the $f$ of the modified $k-\varepsilon$ model is $1.76 \mathrm{H}_{z}$. The modification of the turbulence model seems to improve the shedding frequency by reducing the $f$ values to be nearly similar with the frequency obtained from the experiment of Lyn et al. [38]. The shedding frequency $f$ is associated with a time that is needed to form a vortex behind the square block. It is found that the standard $k-\varepsilon$ turbulence model generates a vortex at every 0.56 seconds and faster than the modification $k$ - $\varepsilon$ turbulence model does, whereby the modified $k-\varepsilon$ model forms a vortex at every 0.57 seconds. The time scale of the eddy turnover is associated with the vortex shedding period, which is expressed as $T_{p}=L / \bar{u}$, where $L$ is the eddy length scale and $\bar{u}$ is the averaged value of the periodic velocity. The relation of $L$ and $\varepsilon$ is defined by $\varepsilon=\bar{u} / L$. An overestimate of $k$ by the standard $k-\varepsilon$ model yields miscalculations of $\varepsilon$ and $L$; as a result, the vortex shedding period will be incorrect [45]. The accuracy of the vortex shedding prediction is also represented with the Strouhal number $\left(S_{t}=f R / U_{o}\right)$, see Table 1 . The modified $k-\varepsilon$ turbulence model gives a relatively similar $S_{t}$ value with that of the experiment of about 0.132 . It seems that the standard $k-\varepsilon$ turbulence model overtimes $S_{t}$ account for about $1.5 \%$. The overprediction of $S_{t}$ is also yielded by the LES and RSE models about $1.5 \%$ and $3.0 \%$, respectively. The comparison of the Strouhal number between the standard $k$ $\varepsilon$ and the modified $k-\varepsilon$ turbulent models proves that the implementation of a vorticity term $\Omega$ to replace one strain stress, $S$, improves the simulation prediction of the vortex shedding flow past a square block. 
Figure 9 presents the non-dimensional time-averaged streamwise velocity along the domain centreline of the two present simulation results and can be used to determine the length of the separation zone or the recirculation zone behind the square block. The numerical simulation results of vortex shedding past a square block by Rodi [20] using the Reynolds stress equation model (RSE) and by Bouris [15] using the large eddy simulation (LES) are also included in Figure 9. The experiment measurement of the vortex shedding is available behind the cube and shows that the rotating flow occurred from $x / R=1$ until 4 , after that $\bar{u} / U_{o}$ is relatively constant at about 0.6 until the end of the tank. Behind the obstacle for $x / R>4$, the magnitude of time-averaged streamwise velocities is reduced by $40 \%$ compared to the free stream input velocity. The vortex street appears behind the block as a result of massive separation and produces a reverse flow and velocity fluctuations. The flow conditions behind the square block lead the momentum to be distributed in any direction. Thus, the velocity in the streamwise direction is reduced.

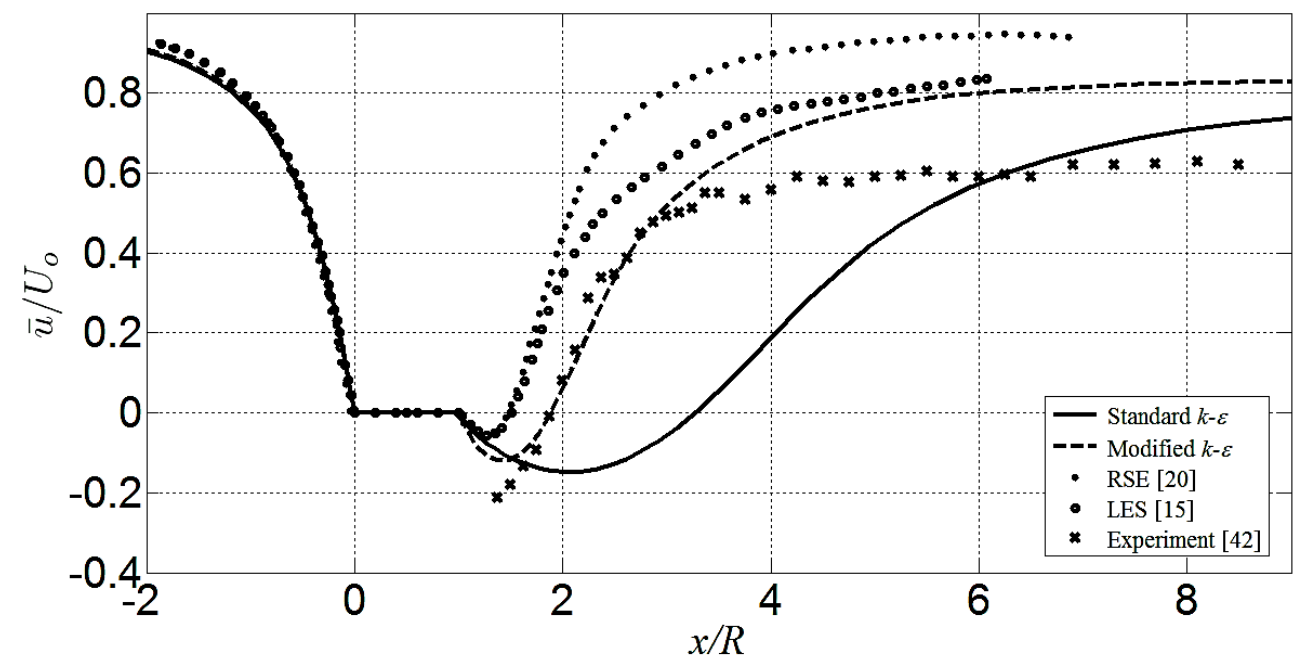

Figure 9. Predicted and experiment measurement of the time averaged streamwise velocity components $\bar{u} / U_{o}$ at $y / R=0.0$

It can be concluded from the simulation results that vortex shredding occurs after the block. The standard $k-\varepsilon$ turbulence model overestimates are about two times larger than the recirculation zone behind the square block compared with the measurement data. It was described in Kato and Launder [37] that the standard $k-\varepsilon$ model leads a weak periodic motion as a consequence of a lack momentum exchanges, resulting in a much longer time-mean separation zone [20]. It can be seen that the rotating flows of the standard $k-\varepsilon$ model, which can be identified by the curve line of stream wise velocity, dominantly occur at $x / R=1$ to 8 . Conversely, the RSE and LES models predict increasingly short recirculation zones, about $35 \%$ smaller than those of the experiment. Even though the LES model shows a better result than the RSE model, the LES overpredicted the streamwise free stream velocity at the downstream by about $35 \%$ compared with that in the experiment. In addition, the difference of the free stream velocities downstream between the results of the RSE model and the experiment measurements is $44 \%$. In contrast, the modified turbulence model provides a relatively similar pattern of the recirculation zone at $x / R=1$ to 3 compared to that of the experiment. The discrepancy of $\bar{u} / U_{o}$ between the simulation results and the experimental data is also identified behind the block at $x / R=1$, where the minimum value of the velocity computed 
by the modified model is $50 \%$ larger than the experiment measurement. Even though the pattern of the time-averaged streamwise velocities calculated with the modified turbulence model at $1<x / R<3$ is in agreement with the data of the experiment measurement, the values $\bar{u} / U_{o}$ of the simulation are 0.2 larger than those of the experiment from $x / R>3$ to the end of the tank. The modified $k-\varepsilon$ model still produces the free stream velocity larger than Lyn's experiment [44]. According to the 3D LES and 2D RANS simulations conducted by Rodi [2], it has been reported that 3D LES is superior over 2D RANS in calculating $\bar{k}$ and $\bar{u}$. The underprediction of the turbulence fluctuation that induces the recovery of the free stream velocity may appear because of the deficiency of 2D RANS to calculate the 3D effect of the turbulent flow. The accuracy of the streamwise time averaged velocity behind the block of the various models, that are compared with the experiment measurement, is also assessed with the root mean square error method (RMSE), where the best value of RMSE is equal to zero, which implies an identical data set between the measurement and the calculation. The modified $k-\varepsilon$ turbulence model provides the best computation result compared with three other models. The RMSE of the modified $k-\varepsilon$ turbulence model is 0.132 and the RMSE values of the standard $k-\varepsilon$ turbulence model, LES model, and RSE model are $0.324,0.195$, and 0.325 , respectively. The RMSE values of the various turbulence models indicate that the modified $k-\varepsilon$ turbulence model is more reliable in computing the velocity fluctuation of the flow around the square block.

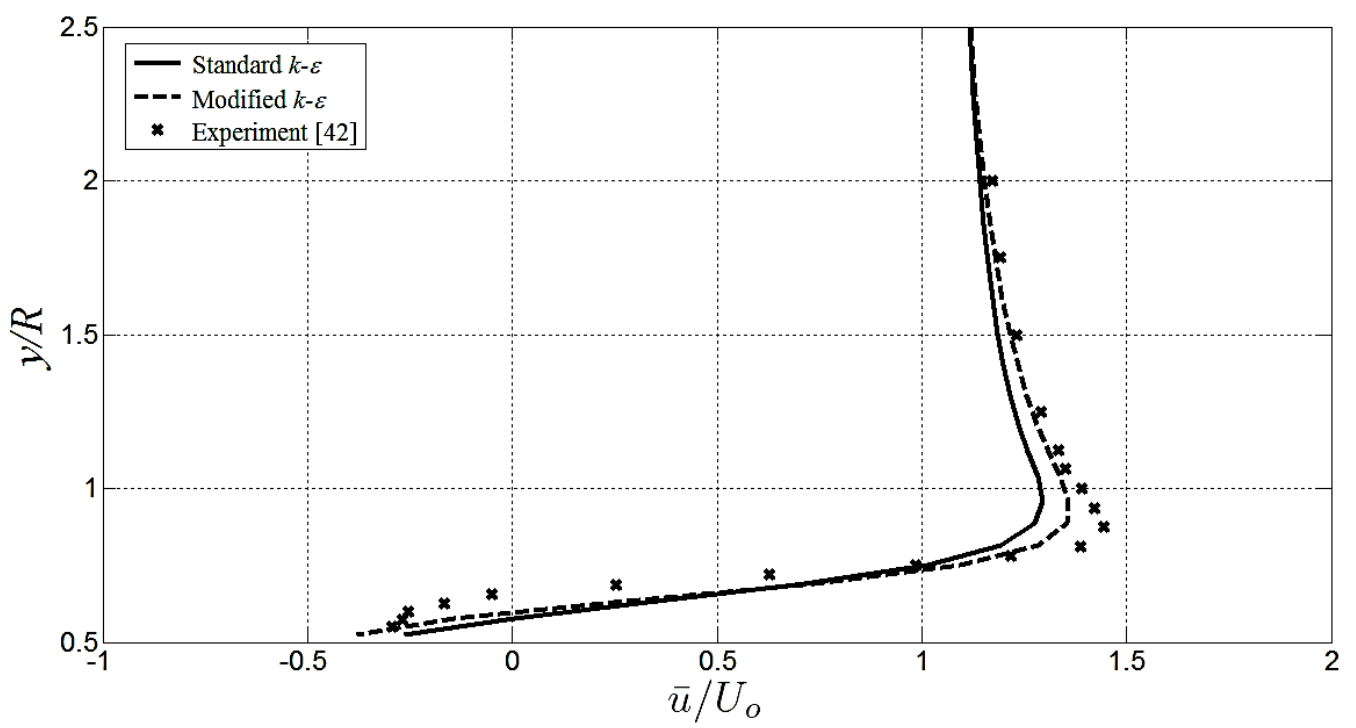

Figure 10.Prediction and experiment of the time averaged streamwise velocity components $\bar{u} / U_{o}$ at $x / R=0.5$.

Figure 10 shows the transverse profile of the non-dimensional time averaged streamwise velocity, $\bar{u} / U_{o}$, above the structure at the $x / R=0.5$. It can be noticed that the boundary layer flow is formed close to the wall of the square block in which there are adverse flows at $0.5<y / R<0.6$. Both computation results present good agreement with the experiment measurement. According to the boundary layer theory, the flow region of the high Reynolds number flow is divided into two layers. In the bulk upper layer, the viscosity can be ignored and the flow can be considered as inviscid outer flow. The second region is a thin layer near the wall, referred to as boundary layer [46]. The shear stress 
adjacent to the structure wall generated by eddy viscosity in the high Reynolds number flow has a retarding effect causing the flow velocity reduction. A continuous retardation leads an adverse flow in the boundary layer. In the boundary layer, the viscous stress force is dominant over the inertia force and its magnitude decreases as the distance from the wall increases. Furthermore, the inertia force is dominant over the viscous stress force in outer layer where the viscous stress force could be neglected and the flow is considered as an inviscid flow or a free stream flow. The accuracy in predicting boundary layer flow depends on the ability of the turbulence model to compute the correct eddy viscosity near to the wall.

The modified $k-\varepsilon$ turbulence model slightly improved the velocity profile. The performances of both standard and modified turbulence $k$ - $\varepsilon$ models are also compared by RMSE. It points out that the modified $k-\varepsilon$ model is better than the standard model when their RMSE values are 0.21 and 0.25 , respectively. The discrepancy between the computation results and the measurement of the velocity occurs near the block surface and vanishes as the measurement points away from the block surface. The slight difference of the velocity profiles of the measurement and the computations can be found mainly from $y / R$ from 0.5 to 1.0 . Near the block wall, the velocity gradient is high. Hence the eddy viscosity computed by the turbulence model is relatively high compared to that in the outer layer. The discrepancy near the wall might be due to the inaccuracy of the turbulence $k-\varepsilon$ models in calculating the eddy viscosity, which significantly affects the strain stress rate in the momentum equation.

\section{CONCLUSIONS}

The modified $k-\varepsilon$ turbulence model is an improved model of the standard $k-\varepsilon$ turbulence model by substituting the one strain stress term $S$ with a vorticity term $\Omega$ and applying a non-constant $C \mu$ equation in computing the production term $P_{k}$ in the turbulent kinetic energy transport equation. Similar with the standard $k-\varepsilon$ turbulence model, the modified $k-\varepsilon$ turbulence model is a stochastic turbulence model that resolves the superimposed random turbulent fluctuation component in the Reynold Averaged Navier-Stokes (RANS) model. In this study, the modified $k-\varepsilon$ turbulence model and the standard $k-\varepsilon$ turbulence model have been simulated with a vortex shedding around a square block case and to maintain the similarity of the condition, both models were set up with a similar flow condition, as well as used an identical computation domain. The results of the simulation indicate that both turbulence models generate vortex shedding behind the square block. The contour plot of the instantaneous turbulent kinetic energy $k$ shows that the standard $k-\varepsilon$ turbulence model produces excessive turbulence kinetic energy in front of the square block and it reduces when the corrected production term $P_{k}$ is applied in the modification model. The instantaneous vorticity contour, the drag coefficient $C_{d}$ fluctuation, and the non-dimensional transverse velocity component $v / U_{o}$ calculated with the modified $k-\varepsilon$ turbulence model show more a vigorous vortex shedding, which indicates an increased momentum transfer to the transverse direction. The modified $k-\varepsilon$ turbulence model has a reduced spin up time from an initial non-turbulent field to a fully developed turbulent flow than the standard $k-\varepsilon$ turbulence model. A reduced spin up time will reduce the CPU time and the simulation would be inexpensive.

Engineering parameters, such as the drag coefficient $C_{d}$ and the Strouhal number $S_{t}$ of the modified $k-\varepsilon$ model indicate improvements whereby $S_{t}$ is relatively similar with that of the experiment and its $C_{d}$ is improved by about $16 \%$ compared to the $C_{d}$ of the standard $k-\varepsilon$ model. Regarding the streamwise time averaged velocity at the centreline of 
the domain, it can be concluded that the modified $k-\varepsilon$ model produces a good agreement with reference to the length of the recirculation zone with the experiment measurement. The standard $k-\varepsilon$ model overpredicted the length of the recirculation zone while the LES model and the RSE model underpredicted the length of the recirculation zones. The time averaged velocity at the centreline behind the block square of the models, which are evaluated by using the RMSE method, indicate that the modified $k-\varepsilon$ model is better than the standard $k-\varepsilon$, LES, and RSE models. The RMSE values of the streamwise velocity in the transverse direction prove that the modified $k-\varepsilon$ model shows a better accuracy in computing the boundary layer profile at the top of the square block. The modified $k-\varepsilon$ turbulence model shows superiority over the standard $k-\varepsilon$ turbulence model. The modified $k-\varepsilon$ turbulence model may produce a more reliable calculation of vortex shedding around a square block. However, the calculation of the downstream velocity of vortex shedding, which has discrepancy with the experiment, needs further improvement.

\section{ACKNOWLEDGEMENTS}

The author would like to thank Faculty of Engineering, Tadulako University for providing the computing facility and the financial support from International Research Collaboration and Scientific Publication Scheme 2017 provided by Directorate Research and Community Services, Ministry of Research, Technology, and Higher Education of Republic Indonesia under contract number 106/SP2H/LT/DPRM/IV/2017.

\section{REFERENCES}

[1] Younis BA, Przulj VP. Computation of turbulent vortex shedding. Comput Mech. 2006;37:408-25.

[2] Rodi W. Comparison of LES and RANS calculations of the flow around bluff bodies. Journal of Wind Engineering and Industrial Aerodynamics. 1997;6971:55-75.

[3] Bosch G, Rodi W. Simulation of vortex a shedding past a square cylinder with different turbulence models. International Journal for Numerical Methods in Fluids. 1998;28:601-16.

[4] Speziale CG, Gatski TB, Sarkar S. On testing models for the pressure-strain correlation of turbulence using direct simulations. Physics of Fluids. 1992;4:288799.

[5] Saha AK, Muralidhar K, Biswas G. Transition and chaos in two-dimensional flow past a square cylinder. Journal of Engineering Mechanics. 2000;126:523-32.

[6] Saha AK, Biswas G, Muralidhar K. Three-dimensional study of flow past a square cylinder at low Reynolds numbers. International Journal of Heat and Fluid Flow. 2003;24:54-66.

[7] Trias FX, Gorobets A, Oliva A. Turbulent flow around a square cylinder at Reynolds number 22,000: A DNS study. Computers \& Fluids. 2015;123:87-98.

[8] Idris MS, Irwan MAM, Ammar NMM. Steady state vortex structure of lid driven flow inside shallow semi-ellipse cavity. Journal of Mechanical Engineering and Sciences. 2012;2:206-16.

[9] Saha AK, Biswas G, Muralidhar K. Numerical study of the turbulent unsteady wake behind a partially enclosed square cylinder using RANS. Computer Methods in Applied Mechanics and Engineering. 1999;178:323-41. 
[10] Shih T-H, Liou WW, Shabbir A, Yang Z, Zhu J. A new $k-\varepsilon$ eddy viscosity model for high Reynolds number turbulent flows. Computers Fluids. 1995;24:227-23.

[11] Wright NG, Easom GJ. Non-linear $k-\varepsilon$ turbulence model results for flow over a building at full-scale. Applied Mathematical Modelling. 2003;27:1013-33.

[12] Li Q, Ma QW, Yan S. Investigations on the Feature of Turbulent Viscosity Associated with Vortex Shedding. Procedia Engineering. 2015;126:73-7.

[13] Elkhoury M. Assessment of turbulence models for the simulation of turbulent flows past bluff bodies. Journal of Wind Engineering and Industrial Aerodynamics. 2016;154:10-20.

[14] Murakami S, Mochida A. On turbulent vortex shedding flow past 2D square cylinder predicted by CFD. Journal of Wind Engineering and Industrial Aerodynamics. 1995;54-55:191-211.

[15] Bouris D, Bergeles G. 2D LES of vortex shedding from a square cylinder. Journal of Wind Engineering and Industrial Aerodynamics. 1999;80:31-46.

[16] Yu D, Kareem A. Numerical simulation of flow around rectangular prism. Journal of Wind Engineering and Industrial Aerodynamics. 1997;67-68:195-208.

[17] Cao Y, Tamura T. Large-eddy simulations of flow past a square cylinder using structured and unstructured grids. Computers \& Fluids. 2016;137:36-54.

[18] Yagmur S, Dogan S, Aksoy MH, Canli E, Ozgoren M. Experimental and numerical investigation of flow structures around cylindrical bluff bodies. In: Petra D, Tomáš V, editors. EPJ Web of Conferences. Český Krumlov, Czech Republic: The European Physical Journal; 2014. p. 02113-p.1--p.7.

[19] Bai W, Mingham CG, Causon DM, Qian L. Detached eddy simulation of turbulent flow around square and circular cylinders on Cartesian cut cells. Ocean Engineering. 2016;117:1-14.

[20] Rodi W. On the simulation of turbulent flow past bluff bodies. Journal of Wind Engineering and Industrial Aerodynamics. 1993;46-47:3-19.

[21] Wilcox DC. Reassessment of the scale-determining equation for advanced turbulence models. AIAA Journal. 1988;26:1299-310.

[22] Tian X, Ong MC, Yang J, Myrhaug D. Unsteady RANS simulations of flow around rectangular cylinders with different aspect ratios. Ocean Engineering. 2013;58:208-16.

[23] Ünal UO, Gören Ö. Effect of vortex generators on the flow around a circular cylinder: computational investigation with two-equation turbulence models. Engineering Applications of Computational Fluid Mechanics. 2014;5:99-116.

[24] Launder BE, Spalding DB. The numerical computation of turbulent flows. Computer Methods in Applied Mechanics and Engineering. 1974;3:269-89.

[25] Kimura I, Hosoda T. A non-linear $k-\varepsilon$ model with realizability for prediction of ows around bluff bodies. International Journal for Numerical Methods in Fluids. 2003;42:813-37.

[26] Sahu SR, Mishra DP. Numerical investigation of maximum air entrainment into cylindrical louvered pipe. International Journal of Automotive and Mechanical Engineering. 2016;13:3278-92.

[27] Wahhad AM, Adam NM, Sapuan SM. Comparison of numerical simulation and experimental study on indoor air quality of air-conditioned office building in desert climate. International Journal of Automotive and Mechanical Engineering. 2015;12:3109-24.

[28] Lam SY, Shuaib NH, Hasini H, Shuaib NA. Computational fluid dynamics investigation on the use of heat shields for thermal management in a car 
underhood. International Journal of Automotive and Mechanical Engineering. 2012;6:785-96.

[29] Najiha MS, Rahman MM, Kamal M, Yusoff AR, Kadirgama K. Minimum quantity lubricant flow analysis in end milling processes: A computational fluid dynamics approach. Journal of Mechanical Engineering and Sciences. 2012;3:340-5.

[30] Najiha MS, Rahman MM. A Computational fluid dynamics analysis of single and three nozzles minimum quantity lubricant flow for milling. International Journal of Automotive and Mechanical Engineering. 2014;10:1891-900.

[31] Norouzi M, Pooladi MA, Mahmoudi M. Numerical investigation of drag reduction in a Class 5 medium duty truck. Journal of Mechanical Engineering and Sciences. 2016;10:2387-400.

[32] Kurnia JC, Sasmito AP. Heat transfer performance of non-circular coiled tubes Research summary, challenges and directions. International Journal of Automotive and Mechanical Engineering. 2016;13:3710-27.

[33] Sana A, Tanaka H. Two-equation turbulence modeling of an oscillatory boundary layer under steep pressure gradient. Canadian Journal of Civil Engineering. 2010;37:648-56.

[34] Norris SE, Richards PJ. Appropriate boundary conditions for computational wind engineering models revisited. The Fifth International Symposium on Computational Wind Engineering. Chapel Hill, North Carolina, USA; 2010.

[35] Park TS, Sung HJ. Development of a near-wall turbulence model and application to jet impingement heat transfer. International Journal of Heat and Fluid Flow. 2001;22:10-8.

[36] Franke R, Rodi W. Calculation of vortex shedding past a square cylinder with various turbulence models. In: Durst F, Friedrich R, Launder BE, Schmidt FW, Schumann U, Whitelaw JH, editors. the Eighth International Symposium on Turbulent Shear Flows. Munich, Germany: Springer; 1991. p. 189-204.

[37] Kato M, Launder BE. The modeling of turbulent flow around stationary and vibrating square cylinders. The ninth international symposium on turbulent shear flows. Kyoto, Japan1993. p. 10.4.1-.4.6.

[38] Lyn DA, Einav S, Rodi W, Park JH. A laser-Doppler velocimetry study of ensemble-averaged characteristics of the turbulent near wake of a square cylinder. Journal of Fluid Mechanics. 1995;304:285-319.

[39] Bosch G, Rodi W. Simulation of vortex shedding past a square cylinder near a wall. International Journal of Heat and Fluid Flow. 1996;17:267-75.

[40] Craft TJ, Launder BE, Suga K. Extending the applicability of eddy viscosity models through the use of deformation invarians. 5th IAHR conference on refined flow modelling and measurement. Paris, France1993. p. 125-32.

[41] CDIT. Research and development of numerical wave channel. Tokyo, Japan: CDIT; 2001.

[42] Isobe M, Xiping Y, Umemua K, Takahashi S. Study on development of numerical wave flume. JSCE Coastal Engineering. Japan: J-STAGE; 1999. p. 36-40.

[43] Amsden AA, Harlow FH. A simplified MAC technique for incompressible fluid flow calculations. Journal of Computational Physics. 1970;6:322-5.

[44] Lyn DA. Vortex shedding past square cylinder. Flows Around Bodies. Manchester: ERCOFTAC; 1992.

[45] Pope SB. Turbulent flows. Cambridge: Cambridge University Press; 2000.

[46] Schlichting H, Gersten K. Boundary-layer theory. 8th ed. Berlin: Springer; 2000. 